Article

\title{
An Efficient Regional Sensitivity Analysis Method Based on Failure Probability with Hybrid Uncertainty
}

\author{
Dawei Zhang ${ }^{1,2}$, Weilin $\mathrm{Li}^{1, *(\mathbb{D})}$, Xiaohua $\mathrm{Wu}^{1}$ and Tie Liu ${ }^{3}$ \\ 1 Department of Electrical Engineering, School of Automation, Northwestern Polytechnical University, \\ Xi'an 710129, Shaanxi, China; hyzdw1979@163.com (D.Z.); wxh@nwpu.edu.cn (X.W.) \\ 2 School of Basic Science for Aviation, Naval Aviation University, Yantai 264001, Shandong, China \\ 3 Coastal Defense College, Naval Aviation University, Yantai 264001, Shandong, China; tieliuyt@sina.com \\ * Correspondence: liweilin907@126.com
}

Received: 7 June 2018; Accepted: 25 June 2018; Published: 27 June 2018

\begin{abstract}
The application of reliability sensitivity analysis (RSA) to the high voltage direct current (HVDC) transmission systems is one of the hot topics in the future. A regional RSA method, the contribution to failure probability (CFP) plot, is investigated in this paper. This CFP plot contains both aleatory and epistemic uncertain variables modeled as random variables by probability theory and interval variables by evidence theory, respectively. A surrogate model of second-level limit state function needs to be established for each joint focal element (JFE), which is a time-consuming process. Additionally, an excessive number of Monte Carlo simulations (MCS) and optimizations may exceed the computing power of modern computers. In order to deal with the above problems and further decrease the computational cost, a more effective CFP calculation method under the framework of random-evidence hybrid reliability analysis is proposed. Three important improvements in the proposed method make the calculation of CFP more efficient and easy to implement. Firstly, an active learning kriging (ALK) based on the symbol prediction idea is employed to directly establish a surrogate model rather than a second-level limit state function with fewer function calls, which greatly simplifies construction of the model. Secondly, a random set-based Monte Carlo simulation (RS-MCS) is used to handle the issue of oversized optimization caused by too many JFEs. Thirdly, for further reducing the size of optimizations and improving the efficiency of the CFP calculation, a Karush-Kuhn-Tucker-based optimization (KKTO) method is recommended in the proposed method to solve the extreme value of performance function. A numerical example and an engineering example were studied to verify the accuracy, effectiveness and practicality of the proposed method. It can be seen from the results that regardless of whether it is modeling or computational efficiency, the proposed method is better than the original method.
\end{abstract}

Keywords: regional sensitivity analysis; Monte Carlo simulation; hybrid reliability analysis; Dempster-Shafer; KKTO

\section{Introduction}

With the advent of AC-DC networking, the reliability of DC systems has become an important factor affecting the reliability of the entire power system. Therefore, evaluating the reliability of the high voltage direct current (HVDC) transmission system has important theoretical significance and practical engineering value. With the increasing level of computational technology and theoretical analysis, progress has been made in the reliability analysis methods for HVDC transmission systems. Reliability sensitivity analysis (RSA) plays an important role in reliability engineering design and risk assessment with the goal of determining how the model inputs affect the model output [1-4]. Generally, RSA can be divided into local reliability sensitivity (LRS) [5-7] and global reliability sensitivity (GRS) 
models [8-10]. LRS focuses on the local effects of the distribution parameters of inputs on the failure probability at their nominal values. Unlike LRS, GRS measures the contribution of the input uncertainty to the model output response by exploring the whole distribution range of input variables. Compared to LRS, GRS has gained more attention in reliability engineering applications during the past few years. Currently, non-parametric [11], variance-based [12], moment independent [13] and entropy-based [14] GRS models have attracted most attention in the research.

All GRS indices can provide the impact level of the input uncertainty on the output response. That is, all GRS indices can identify the important input variables that have a significant impact on the model output. However, they cannot determine which part in the distribution range of input variables has the greatest impact on the output response. In order to deal with this problem and provide better guidance for designers to reduce the output uncertainty, Li et al. [15] proposed a regional RSA technique, the contribution to failure probability (CFP) plot, to analyze the effects of specific regions of input variables containing both aleatory and epistemic uncertainties, to the failure plausibility measure. This CFP plot can be regarded as an extension of the contribution to the sample mean (CSM) plot [16] or contribution to the sample variance (CSV) plot [17]. In Li's study, the aleatory uncertain variables are treated as independent random variables according to probability theory, and the epistemic uncertain variables are modeled by evidence theory with basic probability assignments (BPA) [18-21]. Three steps are required to achieve the CFP plot proposed by Li et al. Firstly, based on evidence theory, the joint focal elements (JFEs) are first obtained. Secondly, based on the non-probabilistic reliability index [22], construct a second-level surrogate model (this model cannot be directly constructed by the relationship between input and output response, which first needs to estimate the non-probabilistic reliability index and treats it as a 'output response' to construct the corresponding surrogate model of limit state function, therefore, the surrogate model constructed by Li et al. is a second-level model) for each JFE according to the corresponding failure plausibility measure. Thirdly, estimate the CFPs of aleatory uncertain variables and epistemic uncertain variables, respectively. This CFP plot not only provides the sensitivity information that the traditional GRS provides, but also measures the contribution of the specific region of model input variable to failure probability, which the traditional GRS cannot provide. That is why there has been growing interest in the CFP recently [23-25].

Although obvious advantages exist in the CFP proposed by Li et al., some shortcomings seriously hinder its application. First, the corresponding surrogate model of second-level limit state function needs to be established for each JFE. This indicates that the number of required surrogate models is equal to the number of JFEs, and it is costly to construct all the required surrogate models when the number of JFEs is very large. Second, it is clear that an efficient modeling method can dramatically reduce the calculation costs of modeling. However, the CFP calculation method does not take into account the number of function calls when establishing a second-level limit state function using the kriging surrogate model. Third, Li et al. pointed out that the non-probabilistic reliability index can be obtained by calling two times the limit state function if the limit state function is monotonic. Otherwise, the optimization method is required. This indicates that it is essential to establish a criterion to screen out those non-monotone intervals to alleviate the computational burden imposed by the optimization method. Unfortunately, $\mathrm{Li}$ et al. does not provide a criterion that can be used to perform this screening task. These three shortcomings seriously prevent the application of the CFP sensitivity analysis method in practical engineering.

In order to improve the computational efficiency of CFP, this paper is dedicated to solving the three tricky issues mentioned above and proposes an efficient CFP calculation method. In the proposed method, the RS-MCS method $[26,27]$ is used to tackle the issue of the number of JFEs caused by too many evidence variables and their corresponding focal elements (FE). More importantly, compared with the original CFP calculation method that requires several surrogate models, the proposed method requires only one surrogate model to satisfy the computational requirements of the CFP plot. Simultaneously, the surrogate model established in this paper is directly constructed based on the relationship of inputs and the output responses of the performance function rather than based 
on the non-probabilistic index, which greatly decreases the difficulty of modeling. Yang et al. [28] proved that only a surrogate model that correctly predicts the sign of limit state function can meet the requirements of random-evidence hybrid reliability analysis. Based on this viewpoint, an extreme value symbol theorem and an expected risk function (ERF) $[29,30]$ are introduced to construct an efficient active learning kriging (ALK) model under the framework of random-evidence hybrid reliability analysis. In order to screen out those JFEs on which the limit state function is monotone, a Karush-Kuhn-Tucker-based optimization (KKTO) method [28] is used in the proposed method to decrease the optimization burden. Through the above improvements, the computational efficiency and the practicality of CFP will dramatically increase.

The rest of this paper is organized as follows. A briefly introduction to random-evidence hybrid reliability analysis and random-evidence hybrid CFP plot is given in Section 2. The RS-MCS and KKTO optimization method are presented in Section 3 to reduce the calculation cost and improve the efficiency of optimization for the CFP plot. In Section 4, based on the extreme value symbol theorem and ERF, an efficient modeling method under the framework of aleatory and epistemic uncertainties is established. Combined with the RS-MCS, the calculation steps of random-evidence hybrid CFP plot are presented in detail. A numerical example and an engineering example are investigated in Section 5 to demonstrate the efficiency and accuracy of the proposed method. The conclusions of this study are drawn in Section 6.

\section{Review of Random-Evidence Hybrid Reliability Analysis and CFP Plot}

\subsection{Fundamental Theory of Random-Evidence Hybrid Reliability Analysis}

Let $X=\left[X_{1}, X_{2}, \cdots, X_{n X}\right]$ denote the random variables describing the aleatory uncertainties of model inputs and modeled by probability theory, $Y=\left[Y_{1}, Y_{2}, \cdots, Y_{n Y}\right]$ represent the epistemic uncertain variables modeled by the evidence theory with BPA, $n X$ and $n Y$ are the number of aleatory uncertain variables and epistemic uncertain variables, respectively. Then, the random-evidence hybrid limit state function can be represented as $G=G(\boldsymbol{X}, \boldsymbol{Y})$. The $j$ th epistemic uncertain variable $Y_{j}$ can be described by evidence space $\left(C_{j}, m_{Y j}\right)$, where $C_{j}=\left\{C_{j}^{1}, C_{j}^{2}, \cdots, C_{j}^{n_{j}}\right\}$ denotes the set of FEs, $n_{j}$ is the number of the FEs of $Y_{j}, m_{Y j}$ represents the corresponding BPA of each FE and $\sum_{i=1}^{n_{j}} m_{{ }_{Y j}}\left(C_{j}^{i}\right)=1$. Then, the evidence space $\left(C_{Y}, m_{Y}\right)$ of epistemic uncertain vector $Y$ can be calculated by Cartesian product method (CPM). Specifically,

$$
\begin{gathered}
C_{\boldsymbol{Y}}=\left\{C_{\boldsymbol{Y}_{1}}, C_{\boldsymbol{Y}_{2}}, \cdots, C_{\boldsymbol{Y}_{n_{Y}}} \mid C_{\boldsymbol{Y}_{k}}=\times_{j=1}^{n Y} C_{j}^{i}, C_{j}^{i} \in C_{j}, k=1,2, \cdots, n_{Y}\right\} \\
m_{Y}\left(C_{\boldsymbol{Y}_{k}}\right)=\prod_{j=1}^{n Y} m_{Y_{j}}\left(C_{j}^{i}\right)
\end{gathered}
$$

where $C_{Y_{k}}$ represents the $k$ th JFE of $C_{Y}$, and the number of JFEs of $Y$ is given by $n_{Y}=\prod_{j=1}^{n Y} n_{j}$. As shown in Table 1, consider $Y$ with two epistemic uncertain variables $Y_{1}$ and $Y_{2}$ containing two and three mutually exclusive FEs, respectively. Obviously, $n_{Y}=2 \times 3=6$ and $\sum_{j=1}^{n_{Y}} m_{Y}\left(C_{Y_{j}}\right)=0.18+0.24+0.18+0.12+0.16+0.12=1$.

\begin{tabular}{|c|c|c|c|}
\hline$Y_{2}$ & $C_{2}^{1}=[0.8,1.0]$ & $C_{2}^{2}=[1.0,1.2]$ & $C_{2}^{3}=[1.2,1.4]$ \\
\hline$Y_{1}$ & $m_{Y 2}\left(C_{2}^{1}\right)=0.3$ & $m_{Y 2}\left(C_{2}^{2}\right)=0.4$ & $m_{Y 2}\left(C_{2}^{3}\right)=0.3$ \\
\hline$C_{1}^{1}=[4.0,4.5]$ & $C_{Y 1}=\left\{C_{1}^{1}, C_{2}^{1}\right\}$ & $C_{Y 2}=\left\{C_{1}^{1}, C_{2}^{2}\right\}$ & $C_{Y 3}=\left\{C_{1}^{1}, C_{2}^{3}\right\}$ \\
\hline$m_{Y 1}\left(C_{1}^{1}\right)=0.6$ & $m_{Y}\left(C_{Y 1}\right)=0.18$ & $m_{Y}\left(C_{Y 2}\right)=0.24$ & $m_{Y}\left(C_{Y 3}\right)=0.18$ \\
\hline$C_{1}^{2}=[4.5,5.0]$ & $C_{Y_{4}}=\left\{C_{1}^{2}, C_{2}^{1}\right\}$ & $C_{Y 5}=\left\{C_{1}^{2}, C_{2}^{2}\right\}$ & $C_{Y 6}=\left\{C_{1}^{2}, C_{2}^{3}\right\}$ \\
\hline$m_{Y 1}\left(C_{1}^{2}\right)=0.4$ & $m_{Y}\left(C_{Y 4}\right)=0.12$ & $m_{Y}\left(C_{Y 5}\right)=0.16$ & $m_{Y}\left(C_{Y 6}\right)=0.12$ \\
\hline
\end{tabular}

Table 1. Joint focal element (JFE) $C_{Y_{j}}$ and corresponding basic probability assignments (BPA) $m_{Y}\left(C_{Y_{j}}\right)$. 
According to the unified reliability analysis with aleatory and epistemic uncertainties proposed by $\mathrm{Du}[31,32]$, the failure probability $P_{f}$ containing both aleatory uncertain variables and epistemic uncertain variables can be expressed as

$$
P_{f}=\sum_{j=1}^{n_{\boldsymbol{Y}}} \operatorname{Pr}\left\{\boldsymbol{Y}_{j} \in C_{\boldsymbol{Y}_{j}}\right\} \operatorname{Pr}\left\{G\left(\boldsymbol{X}, \boldsymbol{Y}_{j}\right)<0 \mid \boldsymbol{Y}_{j} \in C_{\boldsymbol{Y}_{j}}\right\}=\sum_{j=1}^{n_{\boldsymbol{Y}}} m_{\boldsymbol{Y}}\left(C_{\boldsymbol{Y}_{j}}\right) P_{f} \boldsymbol{Y}_{j}
$$

Since $Y_{j}$ can be considered as a vector containing $n Y$ interval variables, $P_{f} \boldsymbol{Y}_{j}=\operatorname{Pr}\left\{G\left(X, Y_{j}\right)<0 \mid Y_{j} \in C_{\boldsymbol{Y}_{j}}\right\}$ has its lower and upper bounds, which are denoted as $P_{f \boldsymbol{Y}_{j}}^{L}$ and $P_{f Y_{j}{ }^{\prime}}^{U}$ respectively. Then, the belief measure $\operatorname{Bel}(F)$ and plausibility measure $P l(F)$ can be calculated as follows according to Equation (3).

$$
\begin{gathered}
\operatorname{Bel}(F)=\sum_{j=1}^{n_{Y}} m_{\boldsymbol{Y}}\left(C_{\boldsymbol{Y}_{j}}\right) P_{f \boldsymbol{Y}_{j}}^{L} \\
\operatorname{Pl}(F)=\sum_{j=1}^{n_{Y}} m_{Y}\left(C_{\boldsymbol{Y}_{j}}\right) P_{f \boldsymbol{Y}_{j}}^{U}
\end{gathered}
$$

where $F=\{\boldsymbol{X}, \boldsymbol{Y} \mid G(\boldsymbol{X}, \boldsymbol{Y})<0\}$.

\subsection{Brief Introduction to Random-Evidence Hybrid CPF Plot}

The random-evidence RSA technique proposed by Li et al. not only can screen out important input variables that have significant impact on $\mathrm{Pl}$, but also determines which part of the input variable has the greatest impact on Pl. According to their work [15], the second-level limit state function of each FE should be firstly established based on the non-probability index. Under the framework of non-probability reliability analysis, for a limit state function, $M(Z)=M\left(Z_{1}, Z_{2}, \cdots, Z_{n Z}\right)$, the non-probability index is given as [33]:

$$
\eta=M^{c} / M^{r}
$$

In general, the larger the non-probability index $\eta$ is, the more reliable the system is. If $\eta>1$ holds, it indicates that the system must be safe. If $\eta<-1$ holds, it indicates that failure will unavoidably happen to the system. If $-1<\eta<1$ holds, whether the system is safe or not is uncertain.

In Equation (6), $M^{c}=\left(M^{U}+M^{L}\right) / 2$ and $M^{r}=\left(M^{U}-M^{L}\right) / 2$ represent the median and deviation of $M$, respectively. $M^{L}$ and $M^{U}$ are the lower bound and upper bound of $M$ and can be calculated as follows:

$$
\begin{aligned}
M^{L} & =\min M\left(Z_{1}, Z_{2}, \cdots, Z_{n Z}\right) \\
M^{U} & =\max M\left(Z_{1}, Z_{2}, \cdots, Z_{n Z}\right)
\end{aligned}
$$

When the system contains both modeled aleatory uncertain variables and epistemic uncertain variables, the non-probability index must be a function of the aleatory uncertain variables $X$, i.e., $\eta=\eta(\boldsymbol{X})$. For a given JFE $C_{\boldsymbol{Y}_{j}}$, the corresponding second-level limit state function based on the $\mathrm{Pl}$ can be established as

$$
M_{\boldsymbol{Y}_{j}}^{(2)}=\eta Y_{Y_{j}}(\boldsymbol{X})-1
$$

By setting the quantile of $X_{i}$ as $q$, the failure plausibility measure for $C_{Y_{j}}$ can be defined as

$$
P_{f_{Y_{j} X_{i}}^{U}}^{U}(q)=\int_{-\infty}^{\infty} \cdots \int_{-\infty}^{\infty} \int_{-\infty}^{F_{X_{i}}^{-1}(q)} \prod_{i=1}^{n_{X}} f_{X_{i}}\left(X_{i}\right) I_{F_{Y_{j}}}(X) \mathrm{d} X_{i} \mathrm{~d} X_{1} \cdots \mathrm{d} X_{i-1} \mathrm{~d} X_{i+1} \cdots \mathrm{d} X_{n_{X}}
$$


where $q \in[0,1], F_{X_{i}}^{-1}(q)$ is the inverse cumulative distribution function $(\mathrm{CDF})$ of $X_{i}$ at $q . I_{F_{Y_{j}}}(X)$ is an indicator function and is expressed as

$$
I_{F_{Y_{j}}}(\boldsymbol{X})= \begin{cases}1 & M_{Y_{j}}^{(2)} \leq 0 \\ 0 & M_{Y_{j}}^{(2)}>0\end{cases}
$$

It can be seen from Equation (10) that the multiple integral is estimated in the range of $[-\infty,+\infty]$ for all aleatory uncertain variables except for $X_{i}$, of which the range is $\left[-\infty, F_{X_{j}}^{-1}(q)\right]$. Then, the CFP for the aleatory uncertain variable $X_{i}$ is expressed as

$$
\begin{aligned}
\operatorname{CFP}_{X_{i}}(q) & =\frac{\sum_{j=1}^{n_{Y}} m_{Y}\left(C_{Y_{j}}\right) P_{f_{j} X_{i}}^{u}(q)}{P l(F)} \\
& =\frac{1}{P l(F)} \sum_{j=1}^{n_{Y}} m_{Y}\left(C_{Y_{j}}\right) \int_{-\infty}^{\infty} \cdots \int_{-\infty}^{\infty} \int_{-\infty}^{F_{X_{i}}^{-1}(q)} \prod_{i=1}^{n X} f_{X_{i}}\left(X_{i}\right) I_{F_{Y_{j}}}(X) \mathrm{d} X_{i} \mathrm{~d} X_{1} \cdots \mathrm{d} X_{i-1} \mathrm{~d} X_{i+1} \cdots \mathrm{d} X_{n_{X}}
\end{aligned}
$$

It can be seen from Equation (12) that the $\mathrm{CFP}_{X_{i}}(q)$ reflects the effect of the part of distribution of random variable $X_{i}$, i.e., $\left[-\infty, F_{X_{i}}^{-1}(q)\right]$, on the $P$ l. For $q_{1}, q_{2}$ of $X_{i}$, assume $0<q_{1}<q_{2}<1$ and $F_{X_{i}}^{-1}\left(q_{1}\right)=X_{i, 1}, F_{X_{i}}^{-1}\left(q_{2}\right)=X_{i, 2}$. The following ratio relationship is established:

$$
v_{X}\left(X_{i, 1}, X_{i, 2}\right)=\frac{P l_{\left[X_{i, 1}, X_{i, 2}\right]}}{P l}=\frac{\mathrm{CFP}_{X_{i}}\left(q_{2}\right)-\mathrm{CFP}_{X_{i}}\left(q_{1}\right)}{\int_{X_{i, 1}}^{X_{i, 2}} f_{X_{i}}\left(X_{i}\right) \mathrm{d} X_{i}}
$$

Since $\int_{X_{i, 1}}^{X_{i, 2}} f_{X_{i}}\left(X_{i}\right) \mathrm{d} X_{i}=F_{X_{i}}\left(X_{i, 2}\right)-F_{X_{i}}\left(X_{i, 1}\right)=q_{2}-q_{1}$ holds, Equation (13) is rewritten as the following formulation in this paper,

$$
v_{X}\left(X_{i, 1}, X_{i, 2}\right)=\frac{\mathrm{CFP}_{X_{i}}\left(q_{2}\right)-\mathrm{CFP}_{X_{i}}\left(q_{1}\right)}{q_{2}-q_{1}}
$$

Equation (14) reflects the reduction of $P l$ due to any reduced uncertain range of $X_{i}$.

When only considering the former $k$ FEs of epistemic uncertain variable $Y_{j}$, the total number of JFEs is $n_{Y_{j_{k}}}=k n_{1} \cdots n_{i-1} n_{i+1} \cdots n_{n_{Y}}$. Denote the $j_{k}$ th JFE of $Y$ and the corresponding BPA as $C_{Y_{j_{k}}}$ and $m_{Y}\left(C_{Y_{j_{k}}}\right)$, respectively. Then, the $P l$, in which the FEs of $Y_{j}$ is reduced to the former $k$ FEs, is expressed as

$$
P l_{Y_{j_{k}}}(F)=\sum_{j_{k}=1}^{n_{Y_{j_{k}}}} m_{\boldsymbol{Y}}\left(C_{Y_{j_{k}}}\right) P_{f Y_{j_{k}}}^{U}
$$

Then, the CFP for $Y_{j}$ with the former $k$ FEs is defined as

$$
\operatorname{CFP}_{Y_{j}}(k)=\frac{P l_{Y_{j_{k}}}(F)}{P l(F)}=\frac{1}{P l(F)} \sum_{j_{k}=1}^{n_{Y_{j_{k}}}} m_{Y}\left(C_{Y_{j_{k}}}\right) P_{f Y_{j_{k}}}^{U}
$$

Assume that for any $k_{1}$ and $k_{2}$ satisfying $0<k_{2}<k_{2}<n_{j}$, the following ratio relationship is established.

$$
v_{Y}\left(Y_{j, k_{1}}, Y_{j, k_{2}}\right)=\frac{P l_{\left[Y_{j, k_{1}}, Y_{j, k_{2}}\right]}}{P l}=\frac{\operatorname{CFP}_{Y_{j}}\left(k_{2}\right)-\operatorname{CFP}_{Y_{j}}\left(k_{1}\right)}{m_{Y}\left(C_{Y_{j_{k_{2}}}}\right)-m_{Y}\left(C_{Y_{j_{k_{1}}}}\right)}
$$

The meanings of Equations (16) and (17) are similar to those of Equations (12) and (14), respectively. Next, the Monte Carlo solution for the CFP plot is briefly represented below, and this CFP is also discussed. 


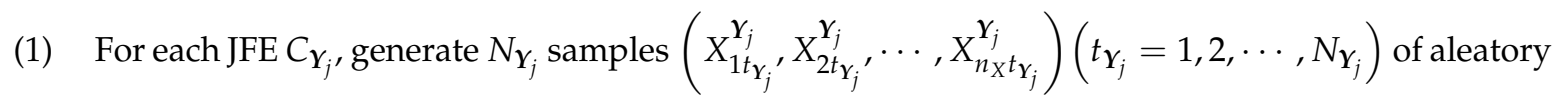
uncertainty variables $X$ according to their joint probability density function (PDF). Based on Equation (9), the corresponding output values $M_{\gamma_{j} t_{\gamma_{j}}}^{(2)}$ are obtained.

(2) Calculate the values $I_{F_{Y_{j}}}$ by Equation (11).

(3) Sort the samples $X_{i t_{Y_{j}}}^{Y_{j}}$ of the random variables $X_{i}$ in ascending order and rename them as $\left(X_{i}^{Y_{j}(1)}, X_{i}^{Y_{j}(2)}, \cdots, X_{i}^{Y_{j}\left(N_{Y_{j}}\right)}\right)$, the corresponding values of the indicator function Equation (11) are represented as $\left(I_{F_{Y_{j}}}^{j(1)}, I_{F_{Y_{j}}}^{j(2)}, \cdots, I_{F_{Y_{j}}}^{j\left(N_{Y_{j}}\right)}\right)$.

(4) The $\mathrm{CFP}_{X_{i}}$ at $q$ quantile for the random variable $X_{i}$ is estimated as follows according to Equation (12):

$$
\mathrm{CFP}_{X_{i}}(q)=\frac{\sum_{j=1}^{n_{Y}} m_{Y}\left(C_{Y_{j}}\right) \sum_{t_{Y_{j}}=1}^{\left|q N_{Y_{j}}\right|} I_{F_{Y_{j}}}^{j\left(N_{Y_{j}}\right)} / N_{Y_{j}}}{\sum_{j=1}^{n_{Y}} m_{Y}\left(C_{Y_{j}}\right) \sum_{t_{Y_{j}}=1}^{N_{Y_{j}}} I_{F_{Y_{j}}}^{j\left(N_{Y_{j}}\right)} / N_{Y_{j}}}, q \in[0,1]
$$

(5) Taking the former $k$ FEs of the aleatory uncertain variable $Y_{j}$, the corresponding $\operatorname{CFP}_{Y_{j}}(k)$ can be given as follows according to Equation (16).

$$
\mathrm{CFP}_{Y_{j}}(k)=\frac{\sum_{j_{k}=1}^{n_{Y_{j_{k}}}} m_{Y}\left(C_{Y_{j_{k}}}\right) \sum_{t_{Y_{j}}=1}^{N_{Y_{j}}} I_{F_{Y_{j}}}^{j\left(N_{Y_{Y_{j}}}\right)} / N_{Y_{j}}}{\sum_{j=1}^{n_{Y}} m_{Y}\left(C_{Y_{j}}\right) \sum_{t_{Y_{j}=1}}^{N_{Y_{j}}} I_{F_{Y_{Y_{j}}}^{j\left(N_{Y_{j}}\right)} / N_{Y_{j}}}^{\left(N_{Y_{j}}\right.}}, 0 \leq k \leq n_{j}
$$

The key to calculating the CFP is to solve the plausibility failure probability, i.e., $P l$. However, according to Equation (5), the calculation of $P l(F)$ is a nested double-loop process. The outer layer calculates the JFEs of $\boldsymbol{Y}$ and their corresponding BPA $m_{Y}\left(C_{Y_{j}}\right)$. The inner layer estimates the extreme value of the second-level limit state function with respect to each JFE by using the optimization method. According to steps (1) and (2), $N_{Y_{j}}$ optimizations are required for each JEF. Therefore, total $N_{Y_{j}} \times n_{Y}=N_{Y_{j}} \times \prod_{j=1}^{n Y} n_{j}$ optimizations are required in step (2) to calculate the extremum of second-level limit state function to determine the value of $I_{F_{Y_{j}}}$. For example, for a model including 5 epistemic uncertain variables with 3 focal elements for each epistemic uncertain variable, the total number of JFEs is $3^{5}=243$, therefore, the construction of such a large-scale surrogate model group is a computationally-intensive and time-consuming process. Moreover, assuming the number of $\boldsymbol{X}$ samples, i.e., $N_{Y_{j}}$, is equal to $10^{6}$ generated in step (1), a total of $243 \times 10^{6}$ optimizations are required in step (2) to calculate the value of $I_{F_{Y_{j}}}$. Such a large optimization is a serious burden for engineering applications. The shortcomings discussed above severely hinder the application of this RSA method. In order to deal with the above issues, a RS-MCS procedure and KKTO method are introduced in the following section, and then an efficient method is proposed for the calculation of CFP plot.

\section{RS-MCS Procedure and KKTO Method}

A new method for the calculation of CFP is proposed based on the RS-MCS procedure. The method can sample aleatory uncertain variables and epistemic uncertain variables at the same time, which eliminates the calculation burden caused by the double-loop process and excessive JFEs. 
A KKTO method is also introduced to further reduce the size of optimization for estimating the extreme value of the limit state function.

\subsection{RS-MCS Procedure}

The Dempster-Shafer structure can be regarded as a closed random set defined in the probability space $[0,1]$ with uniform probability distribution in interval $[26,27]$. Then, the $j$ th epistemic uncertain variable $Y_{j}$ is described by random set as follows,

$$
[0,1] \rightarrow Y_{j}: \alpha_{j} \rightarrow C_{j}^{\alpha_{j}}
$$

where $\alpha_{j}$ is a random variable obeying uniform distribution in interval $[0,1] . C_{j}^{\alpha_{j}}$ represents the corresponding FE mapped by $\alpha_{j}$, which can be obtained by the following rules under the premise of $\sum_{l=1}^{0} m_{Y j}\left(C_{j}^{(l)}\right)=0$.

$$
C_{j}^{\alpha_{j}}=C_{j}^{k} \text { if } \sum_{l=1}^{k-1} m_{Y j}\left(C_{j}^{l}\right)<\alpha_{j} \leq \sum_{l=1}^{k} m_{Y j}\left(C_{j}^{l}\right) l=1,2, \cdots, n_{j}
$$

Then, Equation (4) can be rewritten as the following form through the above mapping relationship,

$$
\begin{aligned}
\operatorname{Bel}(F) & =\sum_{i=1}^{n_{Y}} \operatorname{Pr}\left\{\left(\boldsymbol{X}, C_{\boldsymbol{Y}_{i}}\right) \subseteq F\right\} m\left(C_{\boldsymbol{Y}_{i}}\right) \\
& =\operatorname{Pr}\left\{\boldsymbol{\alpha} \in[0,1]:\left(\boldsymbol{X}, \boldsymbol{C}_{\boldsymbol{Y}_{\boldsymbol{\alpha}}}\right) \subseteq F,\right\}
\end{aligned}
$$

where, $\alpha=\left[\alpha_{1}, \alpha_{2}, \cdots, \alpha_{n_{Y}}\right]$ denotes the vector of all indicator functions; $C_{Y_{\alpha}}=\times_{j}^{n Y} C_{j}^{\alpha_{j}}$ represents the mapped JFE. Then, Equation (22) can be derived as

$$
\begin{aligned}
\operatorname{Bel}(F) & =\iint \cdots \int I\left(\left(\boldsymbol{X}, C_{\boldsymbol{Y}_{\boldsymbol{\alpha}}}\right) \subseteq F\right) f_{\boldsymbol{X}}(\boldsymbol{X}) f_{C_{\boldsymbol{Y}}}(\boldsymbol{\alpha}) d \boldsymbol{X} d \boldsymbol{\alpha} \\
& =E\left[I\left(\left(\boldsymbol{X}, C_{\boldsymbol{Y}_{\boldsymbol{\alpha}}} \subseteq \subseteq\right)\right]\right. \\
& =E\left[I\left(\max _{\boldsymbol{\gamma} \in C_{Y_{\boldsymbol{\alpha}}}}(\boldsymbol{X}, \boldsymbol{Y})<0\right)\right]
\end{aligned}
$$

where $f_{X}(\cdot)$ and $f_{C_{Y}}(\cdot)$ are joint PDF of $X$ and $Y$, respectively. $E(\cdot)$ is the expectation operator. Similarly, Equation (5) can be derived as

$$
\begin{aligned}
P l(F) & =\operatorname{Pr}\left\{\left(\boldsymbol{X}, C_{\boldsymbol{Y}_{\alpha}}\right) \cap F \neq \varnothing\right\} \\
& =E\left[I\left(\left(\boldsymbol{X}, C_{\boldsymbol{Y}_{\alpha}}\right) \cap F \neq \varnothing\right)\right] \\
& =E\left[I \left(\min _{\left.\left.\boldsymbol{Y} \in C_{Y_{\alpha}} G(\boldsymbol{X}, \boldsymbol{Y})<0\right)\right]}\right.\right.
\end{aligned}
$$

Equations (23) and (24) indicate that aleatory uncertain variables and epistemic uncertain variables can be sampled at the same time. Based on the above viewpoints, the MCS procedure of the estimation of CFP is summarized as follows:

(1) Generate $N_{R}$ samples of random variables $X$ according to their joint PDF and denote as $\left\{\boldsymbol{X}^{(s)} \mid s=1,2, \cdots, N_{R}\right\}$.

(2) Generate $N_{R}$ samples of indicator variables $\boldsymbol{\alpha}$ and denoted as $\left\{\boldsymbol{\alpha}^{(s)} \mid s=1,2, \cdots, N_{R}\right\}$. Then obtain their corresponding JFEs according to Equation (21) and denote them as $C_{Y}=\left\{C_{Y}^{(s)} \mid s=1,2, \cdots, N_{R}\right\}$.

(3) According to Equation (24), $P l(F)$ can be estimated by the following equation

$$
P l(F)=\frac{1}{N_{R}} \sum_{s=1}^{N_{R}} I\left(\min _{\gamma \in C_{Y}^{(s)}} G\left(X^{(s)}, Y\right)<0\right)
$$


(4) Sort the samples $X_{i}=\left\{X_{i}^{(s)} \mid s=1,2, \cdots, N_{R}\right\}$ of the random variables $X_{i}$ in ascending order and remark them as $X_{i}^{*}=\left\{X_{i}^{*(s)} \mid s=1,2, \cdots, N_{R}\right\}$, where $X_{i}^{*(s)} \leq X_{i}^{*(s+1)}$. Then, the $\mathrm{CFP}_{X_{i}}$ at $q$ quantile for the aleatory uncertain variable $X_{i}$ can be estimated as follows

$$
\operatorname{CFP}_{X_{i}}(q)=\frac{\sum_{s=1}^{\left|q N_{R}\right|} I\left(\min _{\gamma \in C_{Y}^{(s)}} G\left(X^{*(s)}, \boldsymbol{Y}\right)<0\right) / N_{R}}{\sum_{s=1}^{N_{R}} I\left(\min _{\gamma \in C_{Y}^{(s)}} G\left(\boldsymbol{X}^{(s)}, \boldsymbol{Y}\right)<0\right) / N_{R}}, q \in[0,1]
$$

(5) Sort the sth column of $\boldsymbol{\alpha}$ in ascending order and denote it as $\boldsymbol{\alpha}_{s}^{*}$ and the corresponding JFEs are remarked as $C_{Y_{S}}^{*}$. The number of JFEs containing the former $k$ th FEs of $Y_{j}$ is denoted as $N_{R}^{j(k)}$. Then, the $\mathrm{CFP}_{Y_{j}}$ containing the former $k$ th FEs of $Y_{j}$ can be estimated as follows

$$
\operatorname{CFP}_{Y_{j}}(k)=\frac{\sum_{s=1}^{\left|N_{R}^{j(k)}\right|} I\left(\min _{\boldsymbol{\gamma} \in C_{Y_{i}}^{*(s)}} G\left(\boldsymbol{X}^{(s)}, \boldsymbol{Y}\right)<0\right) / N_{R}}{\sum_{s=1}^{N_{R}} I\left(\min _{\boldsymbol{\gamma} \in C_{Y}^{(s)}} G\left(\boldsymbol{X}^{(s)}, \boldsymbol{Y}\right)<0\right) / N_{R}}, 0 \leq k \leq n_{j}
$$

The number of optimizations to estimate the $P l$ is equal to $N_{R}$. This indicates that the RS-MCS procedure eliminates the computational cost caused by the JFEs and the dimension of evidence variables for the calculation of the CFP plot, which dramatically reduces its computational burden. Thus, it is more efficient compared with the double cycle MCS procedure reviewed in Section 2.2.

However, Equations (26) and (27) still need to repeatedly solve the extremum of the limit state function. The limit state function may be non-monotonous in the entire evidence space, however, it is likely to be monotonous under most JFEs because the interval of each JFE is very small in practical engineering [34]. Since the extreme value occurs at the corners of those 'monotonous' JFEs, it can be directly obtained without additional optimization, which dramatically cuts down the burden of optimization for the extremum estimation. Therefore, it is necessary to propose a more efficient optimization approach that can identify those 'monotonous' JFEs to solve the extremum. Next, a KKTO method is employed to perform this task.

\subsection{KKTO Optimization Method}

Based on Karush-Kuhn-Tucher (KKT) [35], Yang et al. [28] proposed a KKTO optimization method to make the calculation of random-evidence hybrid failure probability more convenient. The KKTO method is briefly introduced in this subsection and used to reduce the optimization costs caused by a large number of simulations. It is noted that the calculation of CFP plot is based on the failure plausibility measure, which only needs to estimate the minimum value of the limit state function. For simplicity, only the process of solving the minimum value of the limit state function is given as follows.

(1) For the $i$ th simulation sample $\left(x^{(i)}, C_{Y}^{(i)}\right)$, the lower bound and upper bound of $C_{Y}^{(i)}$ are denoted as $\underline{y}_{i}$ and $\bar{y}_{i}$, respectively. Then, the minimal vertex point $\underline{y}_{i}^{V}$ is obtained by the following formula

$$
\underline{y}_{i}^{V j}=\left\{\begin{array}{l}
\left.\bar{y}_{i}^{j} \frac{\partial G\left(x^{(i)}, y\right)}{\partial y_{j}}\right|_{y_{j}=y_{i}^{0 j}}<0 \\
\left.\underline{y}_{i}^{j} \frac{\partial G\left(x^{(i)}, y\right)}{\partial y_{j}}\right|_{y_{j}=y_{i}^{0 j}}>0
\end{array}\right.
$$


where $\underline{y}_{i}^{V j}, \bar{y}_{i}^{j}, \underline{y}_{i}^{j}$ and $y_{i}^{0 j}$ are the $j$ th element of the vectors $\underline{y}_{i}^{V}, \bar{y}_{i}^{j}, \underline{y}_{i}^{j}$ and $y_{i}^{0}=\left(\bar{y}_{i}+\underline{y}_{i}\right)$, respectively.

(2) Check whether $\left(x^{(i)}, \underline{y}_{i}^{V}\right)$ meets the following condition

$$
\left\{\begin{array}{l}
H\left(\underline{\boldsymbol{y}}_{i}^{V}\right)=\bigcup_{j=1}^{n_{Y}} H\left(\underline{y}_{i}^{V j}\right) \\
H\left(\underline{\boldsymbol{y}}_{i}^{V}\right)=\left\{\underline{y}_{i}^{V j}=\bar{y}_{i}^{j},\left.\frac{\partial G\left(x^{(i)}, y\right)}{\partial y_{j}}\right|_{y_{j}=\underline{y}_{i}^{V j}} \leq 0\right\} \cup\left\{\underline{y}_{i}^{V j}=\underline{y}_{i}^{j},\left.\frac{\partial G\left(x^{(i)}, y\right)}{\partial y_{j}}\right|_{y_{j}=\underline{y}_{i}^{V j}}>0\right\}
\end{array}\right.
$$

If the above condition holds, the minimum value of the limit state function is $G_{\min }=G\left(x^{(i)}, \underline{y}_{i}^{V}\right)$, otherwise, an optimization algorithm is required to solve $G_{\min }=\min _{y \in C_{Y}^{(i)}} G\left(x^{(i)}, y\right)$.

Note that the sequential quadratic programming (SQP) [36], which is one of the widely used global optimization algorithms, is adopted in the above steps. In addition, the above procedure can be easily executed in parallel and the parfor-loop in MATLAB (2012b-64 bit, MathWorks, Natick, MA, USA) [37].

The key to estimating the CFP is effectively estimating the extreme value of the limit state function by an optimization method, however, the analytic expression of the limit state function cannot be directly obtained in most practical engineering. In order to deal with this issue, an efficient ALK method is recommended in this research for calculating the CFPs that contain both aleatory uncertain variables and epistemic uncertain variables.

\section{Random-Evidence Hybrid Based Active Learning Kriging Model}

\subsection{Basic Idea}

It can be seen from Equations (26) and (27) that the key to estimate the CFP is predicting the sign of the extreme value of the limit state function. Yang et al. proved that a surrogate model that can correctly predict the sign of the limit state function can correctly predict the sign of the extreme value of the limit state function in their research on hybrid reliability analysis involving both aleatory uncertainties and epistemic uncertainties [24], i.e., the following two properties are established.

Property 1. If $\operatorname{sign}(\hat{G}(x, y))=\operatorname{sign}(G(x, y))$, then $\operatorname{sign}\left(\min _{y \in C^{(i)}} \hat{G}(x, y)\right)=\operatorname{sign}\left(\min _{y \in C^{(i)}} G(x, y)\right)$

Property 2. If $\operatorname{sign}(\hat{G}(x, y))=\operatorname{sign}(G(x, y))$, then $\operatorname{sign}\left(\max _{y \in C^{(i)}} \hat{G}(x, y)\right)=\operatorname{sign}\left(\max _{y \in C^{(i)}} G(x, y)\right)$ where $\operatorname{sign}(\cdot)$ is the sign function. From the above two properties it can be seen that although the extreme value solved by a surrogate model may be different with that solved by the original limit state function, the sign of the extreme value predicted by a surrogate model and that of predicted by the original limit state function are identical if the surrogate model can correctly predict the sign of limit state function. Therefore, a surrogate model that can rightly predict the sign of the limit state function (not the specific function value) can satisfy the accuracy requirement of the CFP calculation. An expectation risk function (ERF)-based ALK model is introduced and used to construct a sign prediction surrogate model for limit state function.

\subsection{ERF Based-Active Learning Kriging Surrogate Model}

Suppose $\left[x^{(1)}, x^{(2)}, \ldots, x^{(m)}\right]^{T}$ and $\left[G\left(x^{(1)}\right), G\left(x^{(2)}\right), \ldots, G\left(x^{(m)}\right)\right]^{T}$ are the training samples and the corresponding response values, respectively, used to construct a design of experiments (DoE). Given a DoE, the predict value of $G(x)$ for an unknown sample $x$ provided by kriging model is given as 


$$
\hat{G}(\boldsymbol{x})=\hat{\beta}+\boldsymbol{r}(\boldsymbol{\theta}, \boldsymbol{x})^{T} \mathbb{R}(\boldsymbol{\theta})^{-1}(\boldsymbol{g}-\hat{\beta} \boldsymbol{I})
$$

and the corresponding predicted variance is expressed as

$$
s^{2}(\boldsymbol{x})=\sigma^{2}\left[1+\frac{\left(\boldsymbol{I}^{T} \mathbb{R}^{-1} \boldsymbol{r}(\boldsymbol{\theta}, \boldsymbol{x})-1\right)^{2}}{\boldsymbol{I}^{T} \mathbb{R}^{-1} \boldsymbol{I}}-\boldsymbol{r}^{T}(\boldsymbol{\theta}, \boldsymbol{x}) \mathbb{R}^{-1} \boldsymbol{r}(\boldsymbol{\theta}, \boldsymbol{x})\right]
$$

In Equations (30) and (31), $\boldsymbol{I}$ is an $m$-dimensional unit vector, $\boldsymbol{r}(\boldsymbol{\theta}, \boldsymbol{x})$ and $\mathbb{R}(\boldsymbol{\theta})$ are the correlation vector and correlation matrix, respectively, $g$ denotes the response vector of $x$, i.e., $\left[G\left(x^{(1)}\right), G\left(x^{(2)}\right), \cdots, G\left(x^{(m)}\right)\right]^{T}, \hat{\beta}, \sigma^{2}$ and $\boldsymbol{\theta}$ are model parameters and can be estimated by maximum likelihood estimation. For more detailed information about kriging model refer to [30].

Since the predicted value $\hat{G}(\boldsymbol{x})$ is not the true value of $G(\boldsymbol{x})$ and $G(\boldsymbol{x}) \sim N(\hat{G}(\boldsymbol{x}), s(\boldsymbol{x}))$, there is a risk it will incorrectly predict the sign of the limit state function. In order to measure this risk and add those samples with a large risk of wrong prediction to the training samples to improve the predictive power of the kriging model, an ERF is proposed by Yang et al. and expressed as

$$
E[R(x)]=-\operatorname{sign}(\hat{G}(x)) \hat{G}(x) \Phi\left(-\operatorname{sign}(\hat{G}(x)) \frac{\hat{G}(x)}{s(x)}\right)+s(x) \phi\left(\frac{\hat{G}(x)}{s(x)}\right)
$$

where $\Phi(\cdot)$ and $\phi(\cdot)$ are standard normal CDF and standard normal PDF, respectively. The prediction accuracy of a kriging model for the sign of the limit state function will be greatly improved when adding the sample with the largest ERF to the training samples. With this ERF, the modeling process under the framework of random-evidence hybrid reliability is summarized as follows:

(1) Generate a large quantity of candidate samples and denote as $\Omega$. For a random variable, the candidate samples can be sampled based on its PDF. For an evidence variable, it is first transformed to a random variable using the following uniformity approach $[38,39]$,

$$
f_{Y}\left(y_{j}\right)=\sum_{i=1}^{n_{j}} I\left(y_{j} \in C_{j}^{i}\right) m_{Y j}\left(C_{j}^{i}\right) /\left(\bar{y}_{j}^{i}-\underline{y}_{j}^{i}\right)
$$

where $\bar{y}_{j}^{i}$ and $\underline{y}_{j}^{i}$ are upper bound and lower bound of $i$ th FE of the $j$ th evidence variable. $f_{Y}\left(y_{j}\right)$ can be considered as the PDF of epistemic uncertain variables, then the candidate sample of the evidence variables are generated.

(2) Generate 12 initial training samples and denote them as $\Omega_{0}$. Then, obtain their corresponding response values of limit state function and construct an initial kriging model.

(3) Select a sample with the largest ERF in candidate samples space $\Omega$ based on Equation (32) and denote it as $\left(x^{*}, y^{*}\right)$.

(4) If the selected sample $\left(x^{*}, y^{*}\right)$ satisfies the following convergence criteria, go to step (6). Otherwise, go on.

$$
E\left[R\left(x^{*}, y^{*}\right)\right] / \operatorname{mean}_{\left(x^{i}, y^{i}\right) \in \Omega_{0}}\left|G\left(x^{i}, y^{i}\right)\right| \leq 10^{-4}
$$

(5) Add $\left(x^{*}, y^{*}\right)$ into training samples $\Omega_{0}$ and update the kriging model. Go to step (3)

(6) Exit loop.

Through the above process, a kriging model for predicting the sign of the extreme value of the limit state function is efficiently established. Then, by combining this ALK model with the RS-MCS procedure in Section 3.1 and KKTO optimization method in Section 3.2, an efficient RSA method based on plausibility failure probability under the framework of random-evidence hybrid reliability analysis is proposed. 
It can be seen that the proposed method dramatically reduces the function calls, optimization size and the complexity of surrogate modeling. Next, two examples are studied to illustrate the efficiency and accuracy of the proposed method. Additionally, the MCS, Kirging-MCS, which are the original CFP calculation methods proposed by Li et al., and the RS-MCS employed in this paper are also performed as a reference.

\section{Examples and Discussion}

\subsection{Example 1. Numerical Example}

Consider the following simple equation as a limit state function

$$
G(X, Y)=45-X_{1}^{2}+2 X_{2}-Y_{1} Y_{2}
$$

where $X_{1}$ and $X_{2}$ are aleatory uncertain variables, $Y_{1}$ and $Y_{2}$ are epistemic uncertain variables. The uncertain information of the above two types of input variables are listed in Tables 2 and 3 , respectively.

In this example, the CFP results obtained by the different methods are shown in Figures 1-4. The comparison between four CFP calculation methods illustrates the accuracy and feasibility of the proposed method. From Figures 1-4 it can be seen that the results of the proposed method match well with those of the MCS and RS-MCS. This indicates that the proposed method is correct and has a high accuracy in the calculation of CFP. The number of surrogate models, function calls and the size of optimizations required to complete $P l$ calculation by different methods are listed in Table 4 .

Table 2. Distribution information of aleatory uncertain variables $X$.

\begin{tabular}{cccc}
\hline Variable & Mean & Standard Deviation & Distribution \\
\hline$X_{1}$ & 4 & 0.8 & Normal \\
$X_{2}$ & 6 & 0.6 & Normal \\
\hline
\end{tabular}

Table 3. FEs and corresponding BPA of epistemic uncertain variables $\boldsymbol{Y}$.

\begin{tabular}{ccc}
\hline Variable & FEs & BPA \\
\hline \multirow{3}{*}{$Y_{1}$} & {$[1,1.1]$} & 0.2 \\
& {$[1.1,1.2]$} & 0.4 \\
& {$[1.2,1.3]$} & 0.4 \\
\hline \multirow{2}{*}{$Y_{2}$} & {$[0.5,0.6]$} & 0.1 \\
\cline { 2 - 3 } & {$[0.6,0.7]$} & 0.3 \\
\cline { 2 - 3 } & {$[0.7,0.8]$} & 0.6 \\
\hline
\end{tabular}

Table 4. Comparison between original and proposed the contribution to failure probability (CFP) method.

\begin{tabular}{ccccc}
\hline Methods & $N_{S}$ & $N_{C}$ & $N_{O}$ & $P l(F)$ \\
\hline MCS & - & $>9 \times 10^{6}$ & $9 \times 10^{6}$ & 0.0127 \\
Kriging-MCS & 9 & $9 \times 10^{3}$ & $9 \times 10^{6}$ & 0.0126 \\
RS-MCS & - & $>10^{6}$ & $10^{6}$ & 0.0127 \\
Proposed method & 1 & 53 & 300,034 & 0.0126 \\
\hline
\end{tabular}

In Table $4, N_{S}, N_{C}$ and $N_{O}$ are the number of surrogate models, function calls and the size of optimizations, respectively. In this example, the $\mathrm{Pl}$ estimated by the proposed RS-MCS with $10^{6}$ optimizations is equal to that of the MCS proposed by Li et al. with $9 \times 10^{6}$ (since the number of JFEs 
is $9,10^{6}$ optimizations are required for each FE, therefore, a total of $9 \times 10^{6}$ optimizations are needed for MCS) optimizations. This indicates that the RS-MCS significantly reduces the optimization burden of calculating $\mathrm{Pl}$ without losing the accuracy. Simultaneously, the $\mathrm{Pl}$ obtained by the proposed method and Kriging-MCS are both 0.0126 , and the error is about $0.16 \%$. However, the size of optimizations of the proposed method is 300,034 , which is about $3.33 \%$ of Kriging-MCS with $9 \times 10^{6}$ optimizations. This fully illustrates the efficiency of the proposed method. Furthermore, 9 surrogate models of second-level limit state function are required in the Kriging-MCS method, however, only one surrogate model is needed in the proposed method, which indicates the proposed method significantly simplifies the complexity of modeling. Moreover, the Kriging-MCS method requires $9 \times 10^{3}$ function calls to construct 9 surrogate models, which is a time consuming process (1000 function calls are needed by each JFE to construct the corresponding surrogate model of second-level limit state function). However, the proposed method only requires 53 function calls to establish a surrogate model. This is why the ERF-based ALK modeling approach is recommended in this paper. From the above analysis it can be seen that the complexity of modeling, the size of, and the calculation cost of the proposed method are superior to the Kriging-MCS model originally proposed by Li et al. Next, the region sensitivity results of the numerical examples are discussed in detail as follows, according to Figures 1-4.

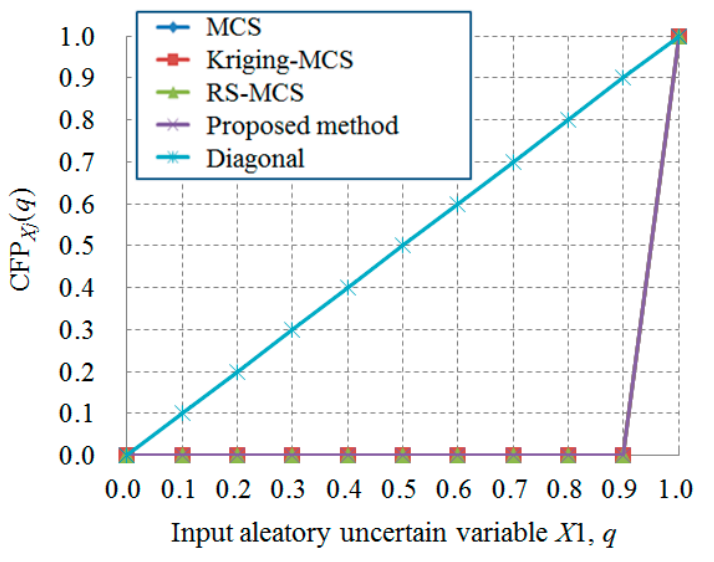

(a)

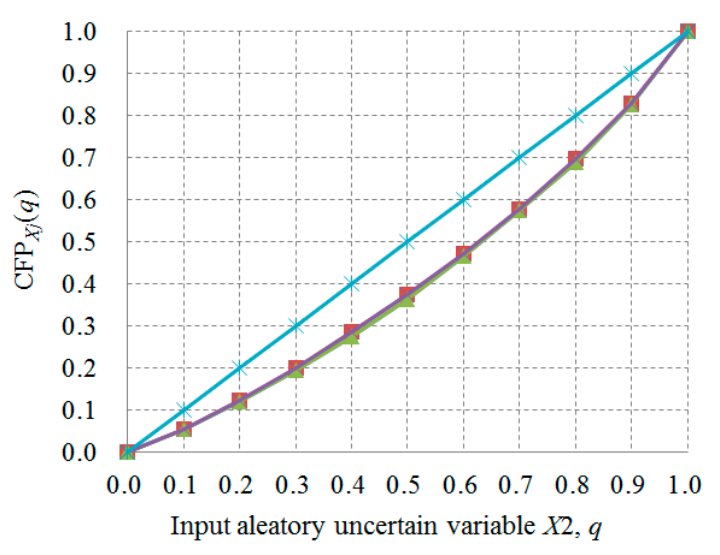

(b)

Figure 1. The $\mathrm{CFP}_{X_{j}}$ plots of the aleatory uncertain variables: (a) The aleatory variables $X_{1}$; (b) The aleatory variables $X_{2}$. CFP: contribution to failure probability; MCS: Monte Carlo simulations.

Figure 1 indicates that the results of the proposed method agree with that of the MCS and RS-MCS method very well. It can be seen from the $\mathrm{CFP}_{X_{j}}$ plots in Figure 1, that $X_{1}$ is more important than $X_{2}$ because the CFP of $X_{1}$ deviates from the diagonal line more than those of $X_{2}$, i.e., $X_{1}$ makes a larger contribution to the failure plausibility measure than $X_{2}$. The above information can also be obtained by a conventional sensitivity analysis method. However, some valuable information that cannot be obtained by the traditional sensitivity analysis method can be obtained by observing the $\mathrm{CFP}_{X_{j}}$ plots. The contributions of the aleatory uncertain variables to $P l$ are not uniform throughout their uncertainty range, so the $P l$ can be effectively reduced by reducing the uncertain ranges of these variables. Only the $10 \%$ values in the right tail of the $X_{1}$ distribution can affect the $P l$. The $20 \%$ values in the right tail of the $\mathrm{X}_{2}$ distribution increase the $\mathrm{Pl}$ by more than $30 \%$. This indicates that the different distribution ranges of aleatory uncertain variables have different effects on the $P l$. In order to clearly show the amount of the $P l$ reduction that would be achieved by reducing the uncertainty range of two aleatory uncertain variables, the $\mathrm{Pl}$ ratio is computed according to Equation (14) and shown in Figure 2. The uncertainty range is divided into 10 intervals for each aleatory uncertain variable in this example. 

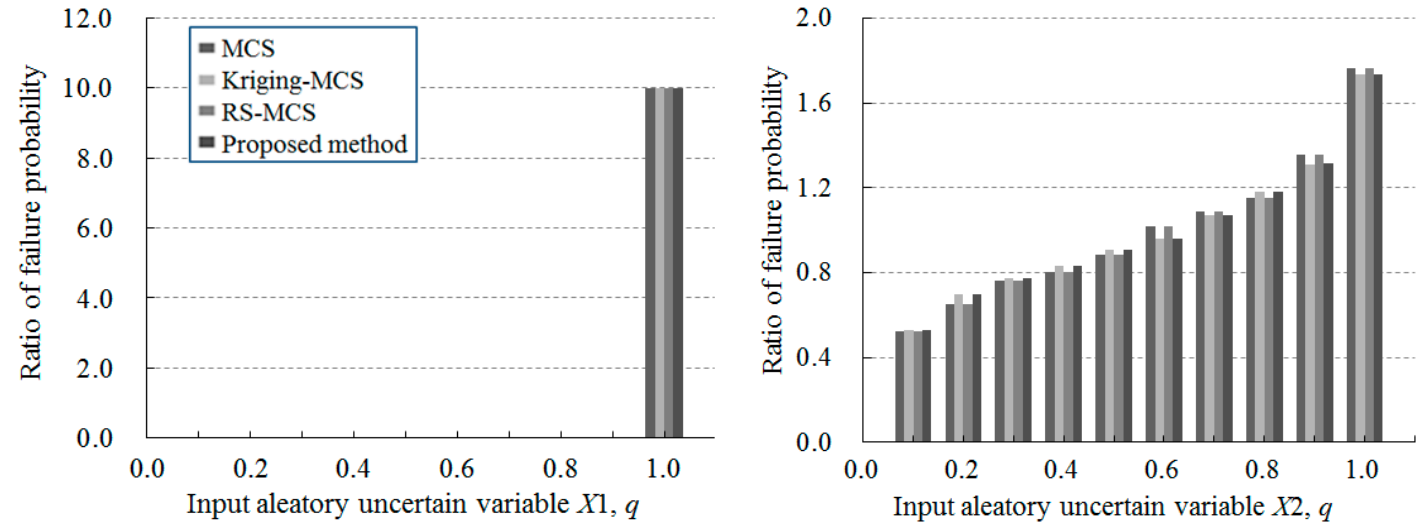

Figure 2. The effect on the system failure plausibility measure by reducing of the range $\left[q_{1}, q_{2}\right]$ of the aleatory uncertain variables.

It can be seen from Figure 2 that the right tail of $X_{1}$, i.e., $\left[F_{X_{1}}^{-1}(0.9), F_{X_{1}}^{-1}(1.0)\right]$ has the most effect on $P l$, which indicates that the $P l$ will be close to zero when reducing the range of $X_{1}$ towards the rest of left interval $\left[F_{X_{1}}^{-1}(0.0), F_{X_{1}}^{-1}(0.9)\right]$. To reduce the $P l$, the range of $X_{1}$ should be reduced towards the left tail of its distribution. This conclusion is also suitable to $X_{2}$.

Figure 3 also illustrates that the results calculated by the proposed method match well with other reference results. It also can be seen that the effect of the evidence variable $Y_{1}$ on $P l$ is similar to that of the evidence variable $Y_{2}$ on $P l$. Moreover, the contributions of the two epistemic uncertain variables to $P l$ are nearly uniform throughout their uncertainty range because the CFPs of $Y_{1}$ and $Y_{2}$ are all closed to the diagonal line. Similar to random variables, the effect of different focal elements within the epistemic uncertain variables on $\mathrm{Pl}$ are explored by Equation (17) and shown in Figure 4.
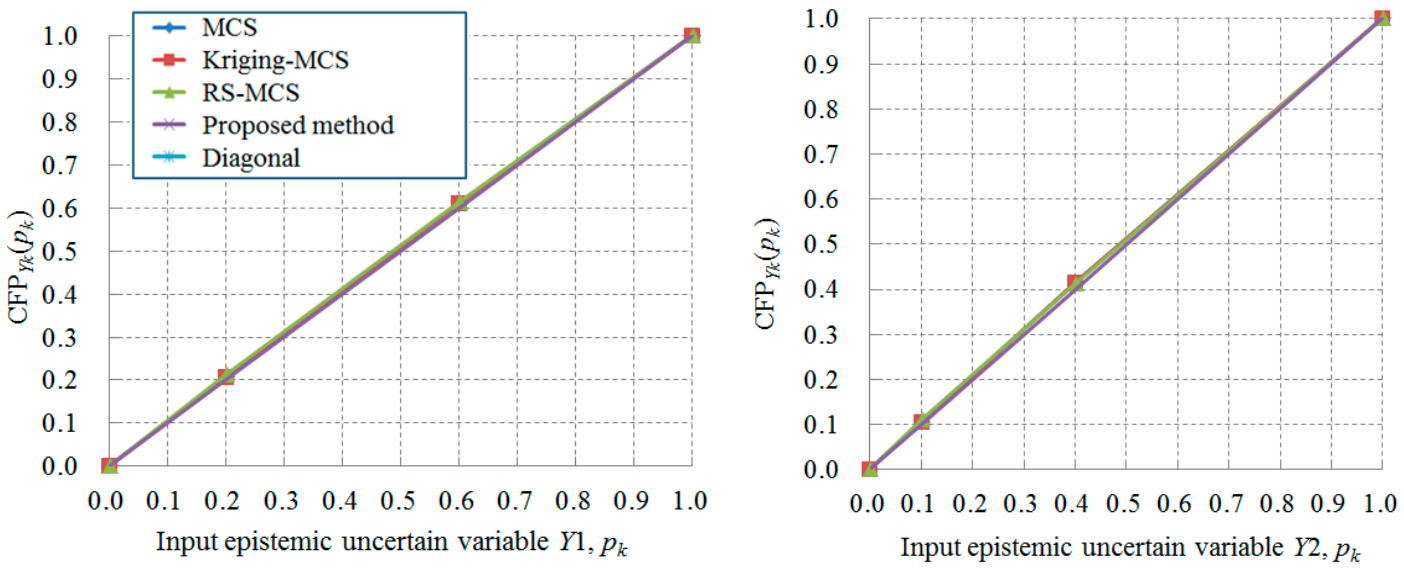

Figure 3. The $\mathrm{CFP}_{Y_{k}}$ plots of the epistemic uncertain variables.

It can be seen from Figure 4 that the effect of each FE of the evidence variable $Y_{1}$ or $Y_{2}$ on $P l$ are similar, therefore, reducing the distribution ranges of $Y_{1}$ and $Y_{2}$ toward any FE will barely affect the $P l$. 

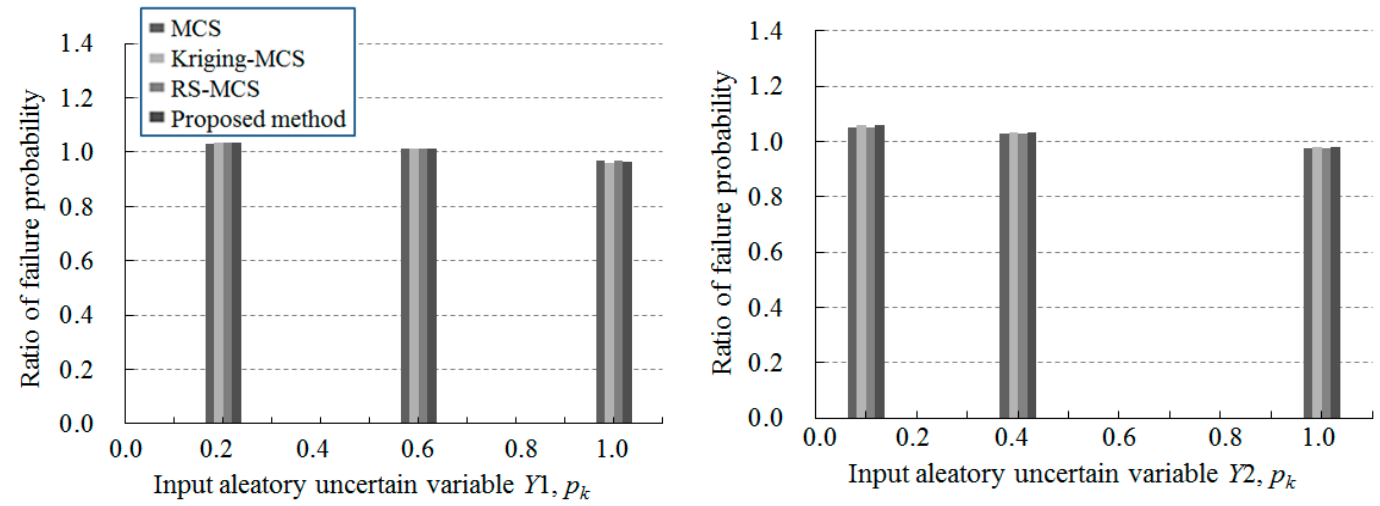

Figure 4. The effect on the system failure plausibility measure by reducing of the range $\left[q_{1}, q_{2}\right]$ of the epistemic uncertain variables.

\subsection{Example 2. A Certain System}

The performance function of a certain system is given as follows,

$$
g(X)=\frac{4 X_{6} X_{5}^{3}}{X_{7} \times\left(X_{3} \times X_{1}^{3}-X_{4} \times X_{2}^{3}\right)}
$$

Assume the threshold of this example is 0.012 , the limit state function can be expressed as follows,

$$
G(X)=0.012-g(X)
$$

The uncertain information of the corresponding aleatory uncertain variables and epistemic uncertain variables are listed in Tables 5 and 6, respectively.

Table 5. Distribution information of aleatory uncertain variables.

\begin{tabular}{cccccc}
\hline Variables & $\boldsymbol{X}_{\mathbf{1}}$ & $\boldsymbol{X}_{\mathbf{2}}$ & $\boldsymbol{X}_{\mathbf{3}}$ & $\boldsymbol{X}_{\mathbf{4}}$ & $\boldsymbol{X}_{\mathbf{7}}$ \\
\hline Mean & 50 & 43 & 35 & 32 & $2 \times 10^{11}$ \\
Standard deviation & 0.05 & 0.043 & 0.035 & 0.032 & $2 \times 10^{8}$ \\
Distribution & Normal & Normal & Normal & Normal & Normal
\end{tabular}

Table 6. FEs and corresponding BPA of epistemic uncertain variables.

\begin{tabular}{ccc}
\hline Variables & FEs & BPA \\
\hline \multirow{3}{*}{$X_{5}$} & {$[980,990]$} & 0.2 \\
& {$[990,1000]$} & 0.6 \\
& {$[1000,1010]$} & 0.2 \\
\hline \multirow{3}{*}{$X_{6}$} & {$[990,995]$} & 0.2 \\
& {$[995,1000]$} & 0.6 \\
& {$[1000,1005]$} & 0.2 \\
\hline
\end{tabular}

In this example, the total number of function calls are 9000 and 59 by the Kriging-MCS proposed by $\mathrm{Li}$ et al. and the proposed method in this paper, respectively. Moreover, the optimization size of the above two methods are $9 \times 10^{5}$ and 35,406, respectively. However, the results of the proposed method match well with that of the MCS or RS-MCS, which can be verified from the comparisons between the results of four methods shown in Figures 5-8. This indicates that the proposed method can efficiently and accurately perform the RSA for the random-evidence hybrid reliability problems in practical engineering. 
It can be seen from Figure 5 that $X_{1}$ has the greatest contribution to the failure plausibility measure because its CFP deviates from the diagonal line most, followed by $X_{2}, X_{3}, X_{4}$ and $X_{7}$. Obviously, the contributions of these aleatory uncertain variables on the $P l$ are not uniform through their whole uncertainty range. About $20 \%$ uncertainty range in the left tail of $X_{1}$ distribution increases the $P l$ by more than $80 \%$. Yet, $20 \%$ uncertainty range in the right tail of $X_{2}$ increases $P l$ by more than $50 \%$. Similar analysis can be performed on the rest of the aleatory uncertain variables.

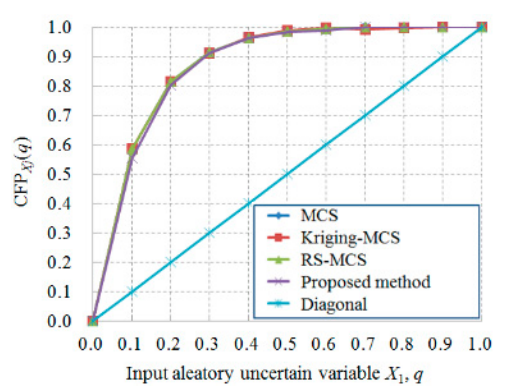

(a)

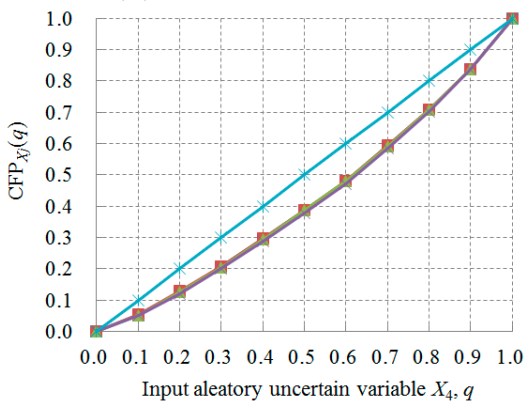

(d)

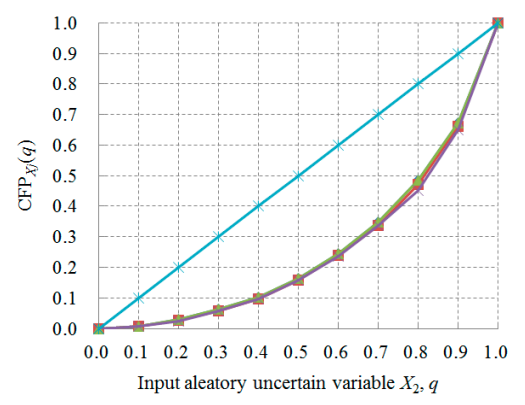

(b)

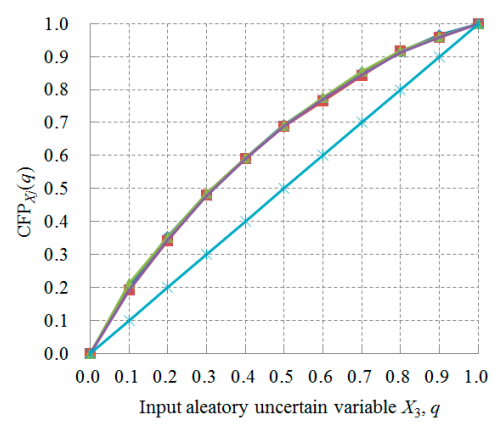

(c)

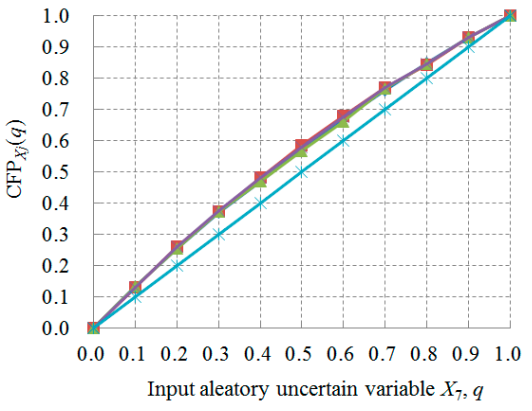

(e)

Figure 5. The $\mathrm{CFP}_{X_{j}}$ plots of the aleatory uncertain variables: (a) The aleatory variables $X_{1}$; (b) The aleatory variables $X_{2}$; (c) The aleatory variables $X_{3}$; (d) The aleatory variables $X_{4}$; (e) The aleatory variables $X_{7}$.

In order to explore the contribution of interval regions of the aleatory uncertain variables to the $P l$, the uncertainty range of each aleatory uncertain variable is divided into 10 intervals. Then, the $P l$ ratio is calculated for each interval, and the corresponding results are shown in Figure 6. It shows that the range $\left[F_{X_{j}}^{-1}(0.0), F_{X_{j}}^{-1}(0.1)\right]$ of the random variable $X_{1}$ has the most effect on the $P l$. Reducing the range of $X_{1}$ towards the right tail of its distribution, the reduction of $P l$ is obvious. If fixing the range of $X_{1}$ at the right side of interval $\left[F_{X_{j}}^{-1}(0.5), F_{X_{j}}^{-1}(0.6)\right]$, i.e., $\left[F_{X_{j}}^{-1}(0.6), F_{X_{j}}^{-1}(1.0)\right]$, the $P l$ will be close to zero. Similar, for reducing the $P l$, the uncertain range of $X_{2}, X_{3}, X_{4}$ and $X_{7}$ can be reduced toward the left, right, right and right side of their mean values $(q \in[0.4,0.5])$, respectively.
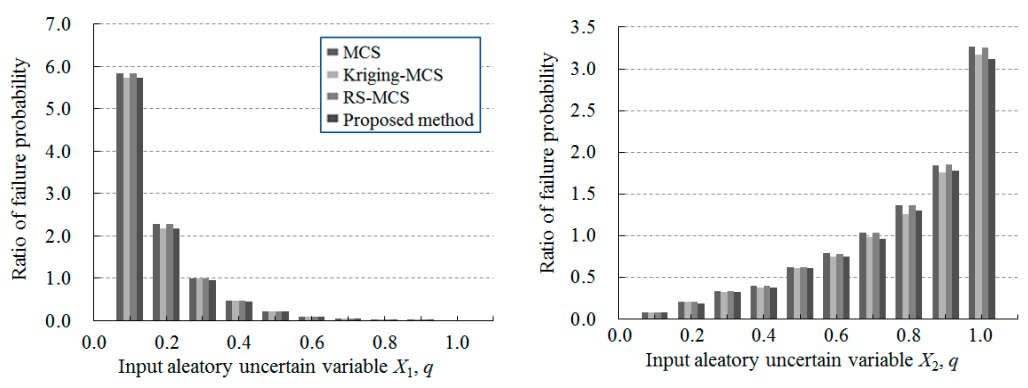

Figure 6. Cont. 

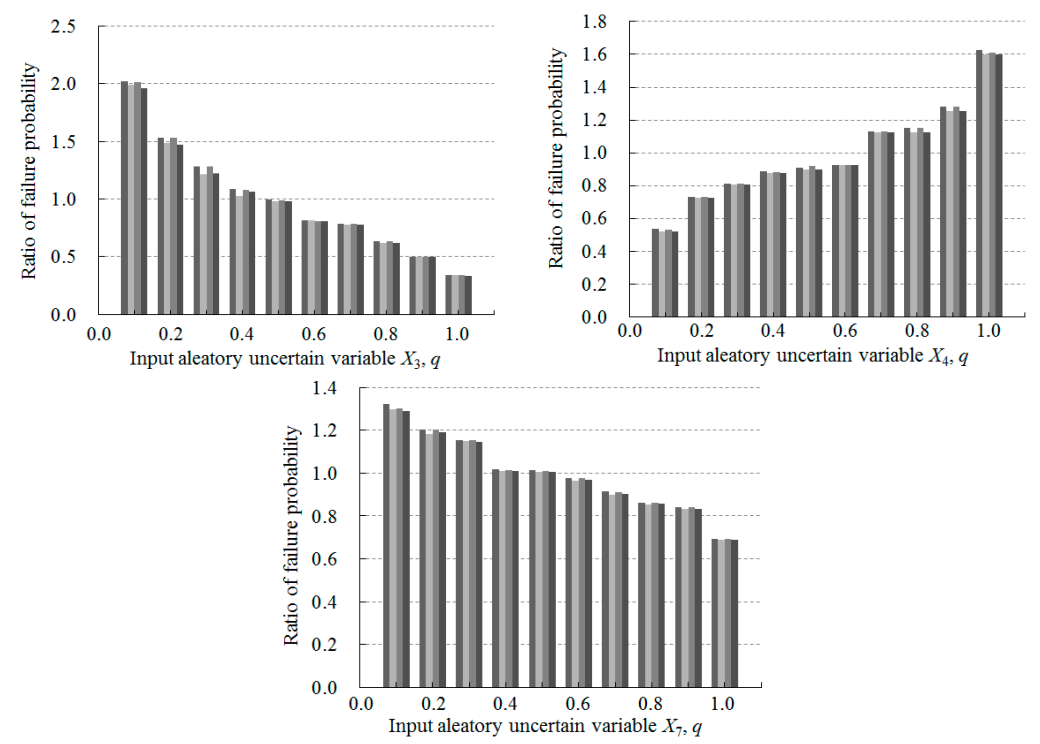

Figure 6 . The effect on the system failure plausibility measure by reducing the range $\left[q_{1}, q_{2}\right]$ of the aleatory uncertain variables.

It can be seen from Figure 7 that the effect of the epistemic uncertain variable $X_{5}$ on the $P l$ is larger than that of the variable $X_{6}$ on $P l$. It also can be seen from Figure 8 that the interval $[1000,1010]$ of $X_{5}$ has the largest effect on $P l$. Additionally, reducing the uncertainty range of $X_{5}$ to [980, 990], [990, 1000] and $[1000,1010]$, the $P l$ will reduce by about $50 \%, 8 \%$ and increase $58 \%$, respectively. However, reducing the uncertainty range of $X_{6}$ to any of its subintervals barely affects the $P l$ because the ratio of failure probability of each JE of $X_{6}$ is close.
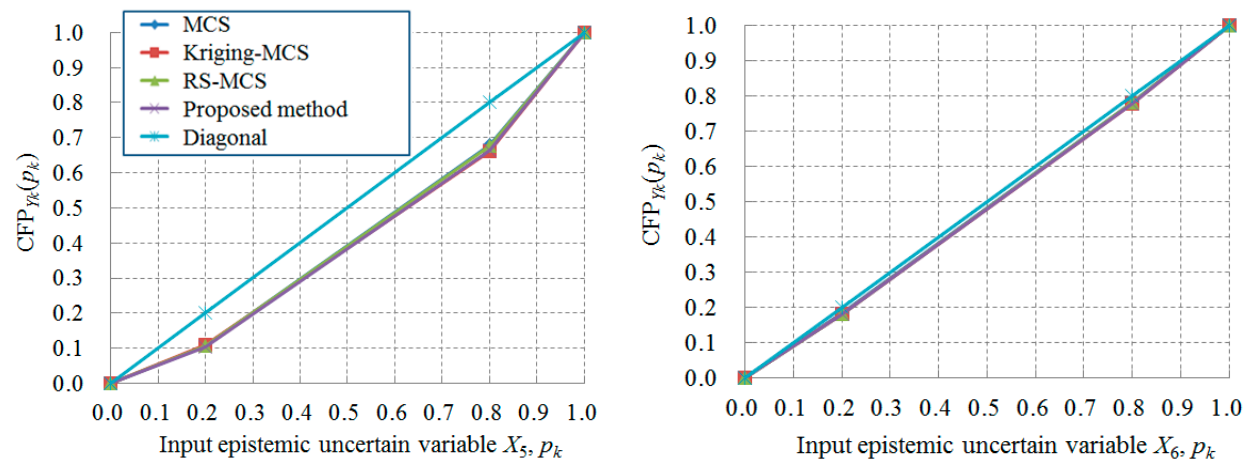

Figure 7. The $\mathrm{CFP}_{Y_{k}}$ plots of the epistemic uncertain variables.
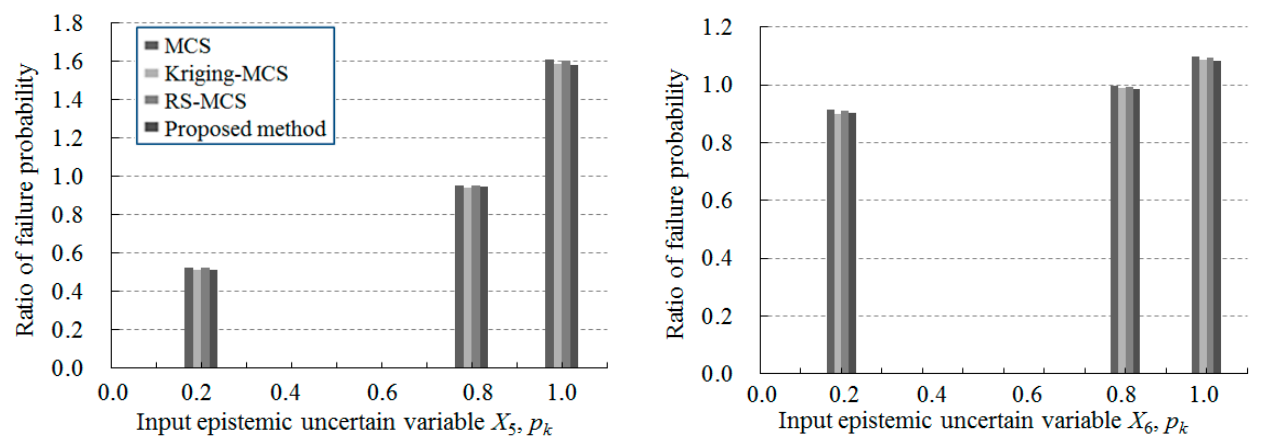

Figure 8 . The effect on the system failure plausibility measure by reducing the range $\left[q_{1}, q_{2}\right]$ of the epistemic uncertain variables. 
The above two examples fully illustrate that the proposed RSA method can not only correctly identify the important aleatory uncertain variables and epistemic uncertain variables or their important interval regions, but also efficiently calculate the CFP without losing too much accuracy. It can be seen that there are two points which illustrate the effective performance of the proposed method. Firstly, only one surrogate model is required by the proposed method to complete the sensitivity analysis. However, the original method requires a set of surrogate models. Secondly, in general, the focus elements are small in practical engineering problems, therefore, the extreme values are likely to occur at the vertices of their focus elements. This point is not noticed in the original method. However, the proposed method makes up for this shortcoming and improves the computational efficiency. Therefore, the RSA can be more effectively calculated by the proposed method.

\section{Conclusions}

A more efficient RSA technique is proposed to measure the contribution of specific uncertain regions of the input random and evidence variables to the failure plausibility measure. The proposed method is based on the RS-MCS method, which eliminates the inconvenience of constructing the second-level limit state function and the burden of too many JFEs that exist in the original CFP technique proposed by $\mathrm{Li}$ et al. Simultaneously, the REF active learning function is used for the construction of a surrogate model to reduce the function calls. Additionally, the KKTO is used to perform the optimization analysis to reduce the burden of optimization. Since the proposed method is not affected by the variable dimension or the number of JFEs and requires only one surrogate model, it can be efficiently applied in practical engineering problems.

A numerical example and a certain system were studied and discussed. The MCS and Kriging-based proposed by Li et al. is also performed in this paper and the results are used as references to illustrate the efficiency and accuracy of the proposed method. The results show that the proposed method can efficiently perform the CFP calculation without losing too much accuracy when compared with MCS and RS-MCS. Additionally, with respect to the complexity of modeling, the size of optimizations or calculation cost, the proposed method is superior to the original Kriging-MCS method.

The proposed method is used to calculate the CFP sensitivity of engineering problems involving random-evidence uncertainties, which can be extended to any type of aleatory-epistemic hybrid uncertainties, such as random-interval hybrid uncertainties, random-convex set hybrid uncertainties, etc. Although the proposed method has high efficiency, there are two small limitations. Firstly, for some complex engineering problems, especially when these problems require commercial software for calculations, because of the need to use time-consuming commercial software to construct a surrogate model, the proposed method will be inefficient. Secondly, when engineering problems involve a large number of evidence variables, or more focus elements exist in each evidence variables, the number of joint focus elements is very large, which reduces the efficiency of the proposed method. However, this is also a problem which is difficult to solve at present, and it is also a research direction in the future.

Author Contributions: D.Z. and X.W. developed the concept and designed the study. Investigation, writing and analysis were done by D.Z. Methodology was done by D.Z. and W.L. Data curation was done by T.L. The manuscript was proofread and revised by all the authors. All of the authors read and approved the final manuscript.

Acknowledgments: This study was supported in part by the National Natural Science Foundation of China (Grant No. 51407144, 51777169), in part by the Aeronautical Science Foundation of China (Grant No. 20164053029), in part by the Fundamental Research Funds for the Central Universities (Grant No. 3102017ZY027, 3102017GX08001), in part by the Young Elite Scientist Sponsorship Program by CAST (2015QNRC001).

Conflicts of Interest: The authors declare no conflict of interest. 


\section{References}

1. Lu, Z.; Song, S.; Yue, Z.; Wang, J. Reliability sensitivity method by line sampling. Struct. Saf. 2008, 30, 517-532. [CrossRef]

2. Cui, L.; Lu, Z.; Wang, Q. Parametric sensitivity analysis of the importance measure. Mech. Syst. Signal Process. 2012, 28, 482-491. [CrossRef]

3. Song, S.; Lu, Z.; Qiao, H. Subset simulation for structural reliability sensitivity analysis. Reliab. Eng. Syst. Saf. 2009, 94, 658-665. [CrossRef]

4. Christen, J.L.; Ichchou, M.; Troclet, B.; Bareile, O.; Ouisse, M. Global sensitivity analysis and uncertainties in SEA models of vibroacoustic systems. Mech. Syst. Signal Process. 2017, 90, 365-377. [CrossRef]

5. Helton, J.C.; Johnson, J.D.; Sallaberry, C.J.; Storlie, C.B. Survey of sampling-based methods for uncertainty and sensitivity analysis. Reliab. Eng. Syst. Saf. 2006, 91, 1175-1209. [CrossRef]

6. Rakovec, O.; Hill, M.C.; Clark, M.P.; Weerts, A.H.; Teuling, A.J. Distributed Evaluation of Local Sensitivity Analysis (DELSA), with application to hydrologic models. Water Resour. Res. 2014, 50, 409-426. [CrossRef]

7. Zhang, F.; Lu, Z.; Cui, L.; Song, S. Reliability sensitivity algorithm based on stratified importance sampling method for multiple failure modes systems. Chin. J. Aeronaut. 2010, 23, 660-669.

8. Sudret, B. Global sensitivity analysis using polynomial chaos expansions. Reliab. Eng. Syst. Saf. 2008, 93, 964-979. [CrossRef]

9. Wei, P.; Lu, Z.; Hao, W.; Wang, B. Efficient sampling methods for global reliability sensitivity analysis. Comput. Phys. Commun. 2012, 183, 1728-1743. [CrossRef]

10. Zhou, C.; Lu, Z.; Li, L.; Feng, J.; Wang, B. A new algorithm for variance based importance analysis of models with correlated inputs. Appl. Math. Model. 2013, 37, 864-875. [CrossRef]

11. Saltelli, A.; Marivoet, J. Non-parametric statistics in sensitivity analysis for model output: A comparison of selected techniques. Reliab. Eng. Syst. Saf. 1990, 28, 229-253. [CrossRef]

12. Saltelli, A.; Annoni, P.; Azzini, I.; Campolongo, F.; Ratto, M.; Tarantola, S. Variance based sensitivity analysis of model output. Design and estimator for the total sensitivity index. Comput. Phys. Commun. 2010, 181, 259-270. [CrossRef]

13. Borgonovo, E.; Castaings, W.; Tarantola, S. Moment independent importance measures: New results and analytical test cases. Risk Anal. 2011, 31, 404-428. [CrossRef] [PubMed]

14. Tang, Z.; Lu, Z.; Jiang, B.; Pan, W.; Zhang, F. Entropy-based importance measure for uncertain model inputs. AIAA J. 2013, 51, 2319-2334.

15. Li, G.; Lu, Z.; Lu, Z.; Xu, J. Regional sensitivity analysis of aleatory and epistemic uncertainties on failure probability. Mech. Syst. Signal Process. 2014, 46, 209-226. [CrossRef]

16. Bolado-Lavin, R.; Castaings, W.; Tarantola, S. Contribution to the sample mean plot for graphical and numerical sensitivity analysis. Reliab. Eng. Syst. Saf. 2009, 94, 1041-1049. [CrossRef]

17. Tarantola, S.; Kopustinskas, V.; Bolado-Lavin, R.; Kaliatka, A.; Ušpuras, E.; Vaišnoras, M. Sensitivity analysis using contribution to sample variance plot: Application to a water hammer model. Int. J. Nonlinear Sci. Numer. Simul. 2015, 99, 62-73. [CrossRef]

18. Ferson, S.; Kreinovick, V.; Ginzburg, L.; Sentz, F. Constructing Probability Boxes and Dempster-Shafer Structures; Technical Report; Sandia National Laboratories: Albuquerque, NM, USA, 2003.

19. Sentz, K.; Ferson, S. Combination of Evidence in Dempster-Shafer Theory; Sandia National Laboratories: Albuquerque, NM, USA, 2002.

20. Ferson, S.; Nelsen, R.B.; Hajagos, J.; Berleant, D.J.; Zhang, J.; Tucker, W.T.; Ginzburg, L.R.; Oberkampf, W.L. Dependence in Probabilistic Modeling, Dempster-Shafer Theory, and Probability Bounds Analysis; Report No. SAND2004-3072; Sandia National Laboratories: Albuquerque, NM, USA, 2004.

21. Tonon, F. Using random set theory to propagate epistemic uncertainty through a mechanical system. Reliab. Eng. Syst. Saf. 2004, 85, 169-181. [CrossRef]

22. Guo, S.; Zhen, L.U.; Feng, Y. A non-probabilistic model of structural reliability based on interval analysis. Chin. J. Comput. Mech. 2001, 18, 56-60.

23. Li, L.; Lu, Z.; Hu, J.X. A new kind of regional importance measure of the input variable and its state dependent parameter solution. Reliab. Eng. Syst. Saf. 2014, 128, 1-16. [CrossRef]

24. Wei, P.; Lu, Z.; Wu, D.; Zhou, C. Moment-independent regional sensitivity analysis: Application to an environmental model. Environ. Model. Softw. 2013, 47, 55-63. [CrossRef] 
25. Zhang, L.; Lu, Z.; Cheng, L.; Hong, D. Moment-independent regional sensitivity analysis of complicated models with great efficiency. Int. J. Numer. Methods Eng. 2015, 103, 996-1014. [CrossRef]

26. Oberguggenberger, M.; Fellin, W. Reliability bounds through random sets: Non-parametric methods and geotechnical applications. Comput. Struct. 2008, 86, 1093-1101. [CrossRef]

27. Alvarez, D.A. On the calculation of the bounds of probability of events using infinite random sets. Int. J. Approx. Reason. 2006, 43, 241-267. [CrossRef]

28. Yang, X.; Liu, Y.; Gao, Y. Unified reliability analysis by active learning Kriging model combining with random-set based Monte Carlo simulation method. Int. J. Numer. Methods Eng. 2016, 108, 1343-1361. [CrossRef]

29. Yang, X.; Liu, Y.; Zhang, Y.; Yue, Z. Probability and convex set hybrid reliability analysis based on active learning Kriging model. Appl. Math. Model. 2015, 39, 3954-3971. [CrossRef]

30. Yang, X.; Liu, Y.; Gao, Y.; Zhang, Y.; Gao, Z. An active learning Kriging model for hybrid reliability analysis with both random and interval variables. Struct. Multidiscip. Optim. 2015, 51, 1003-1016. [CrossRef]

31. Du, X. Uncertainty analysis with probability and evidence theories. In Proceedings of the ASME 2006 International Design Engineering Technical Conferences and Computers and Information in Engineering Conference, Philadelphia, PA, USA, 10-13 September 2006; pp. 1025-1038.

32. Du, X. Unified uncertainty analysis by the first order reliability method. J. Mech. Des. 2008, 130, 091401. [CrossRef]

33. Li, G.; Lu, Z.; Tian, L.; Xu, J. The importance measure on the non-probabilistic reliability index of uncertain structures. Proc. Inst. Mech. Eng. Part O J. Risk Reliab. 2013, 227, 651-661. [CrossRef]

34. Yang, X.; Liu, Y.; Ma, P. Structural reliability analysis under evidence theory using the active learning kriging model. Eng. Optim. 2017, 49, 1922-1938. [CrossRef]

35. Kuhn, H.W.; Tucker, A.W. Nonlinear Programming. In Berkeley Symposium on Mathematical Statistics and Probability; University of California Press: Berkeley, CA, USA, 1951; pp. 481-492.

36. Gill, P.E.; Murray, W.; Saunders, M.A. SNOPT: An SQP algorithm for large-scale constrained optimization. SIAM Rev. 2005, 47, 99-131. [CrossRef]

37. Sharma, G.; Martin, J. MATLAB ${ }^{\circledR}$ : A language for parallel computing. Int. J. Parallel Programm. 2009, 37, 3-36. [CrossRef]

38. Zhang, Z.; Jiang, C.; Wang, G.G.; Han, X. First and second order approximate reliability analysis methods using evidence theory. Reliab. Eng. Syst. Saf. 2015, 137, 40-49. [CrossRef]

39. Jiang, C.; Zhang, Z.; Han, X.; Liu, J. A novel evidence-theory-based reliability analysis method for structures with epistemic uncertainty. Comput. Struct. 2013, 19, 1-12. [CrossRef] 